\title{
Construction and Validation of a Prognostic Model for the Assessment of Postoperative Overall Survival of Patients with Metaplastic Breast Cancer: Based on a Retrospective Large Data Analysis and Chinese Multicenter Study
}

\section{Ge Wang}

Clinical Medical Colleges, Weifang Medical University,Weifang 261000,P.R.China.

\section{Xin Ren}

Clinical Medical college, Weifang Medical University,Weifang261000,Shandong,P.R.China

\section{Mengmeng Wang}

Clinical Medical Colleges, Weifang Medical University,Weifang 261000,P.R.China

Xiaomin Sun

Clinical Medical Colleges, Weifang Medical University,Weifang261000,Shandong,P.R.China.

\section{Yongsheng Wang}

Shandong Cancer Hospital and Institute,Shandong First Medical University and Shandong Academy of Medical Sciences,Jinan 250000,Shandong,P.R.China.

\section{Shukun Zhang}

Department of Pathology, Weihai Municipal Hospital,Cheeloo College of Medicine Weihai 264200,Shandong University.

Jingye Li

Department of Oncology, Linyi Central Hospital,Linyi276000,Shandong,P.R.China.

\section{Wenping Lu}

Department of Oncology, Guang'anmen Hospital,China Academy of Chinese Medicine Sciences,Beijing100053,China

\section{Baogang Zhang}

Department of Pathology, Weifang Medical University,Weifang261053,Shandong,P.R.China.

\section{Pingping Chen}

Department of Pathology, the people's hospital of Rizhao,Rizhao276800,Shandong,P.R.China.

\section{Zhiqiang Shi}

Shandong Cancer Hospital and Institute, Shandong First Medical University and Shandong Academy of Medical Sciences ,Jinan 250000,Shandong,P.R.China.

\section{Lijuan Liu}

Department of Oncology, Weifang Traditional Chinese Hospital,Weifang261000,Shandong.P.R.China. 


\section{Changgang Sun ( $\nabla$ scgdoctor@126.com )}

Department of Oncology, Weifang Traditional Chinese Hospital,Weifang 261000,Shandong,P.R.China. Qingdao Academy of Chinese Medical Sciences,Shandong University of Traditional Chinese Medicine,Qingdao266112,Shandong,P.R.China. https://orcid.org/0000-0002-6648-3602 Jing Zhuang

Department of Oncology, Weifang Traditional Chinese Hospital,Weifang261000,Shandong,P.R.China.

\section{Research Article}

Keywords: metaplastic breast cancer, nomogram, overall survival, postoperative, multicenter

Posted Date: March 15th, 2021

DOl: https://doi.org/10.21203/rs.3.rs-305440/v1

License: (c) (i) This work is licensed under a Creative Commons Attribution 4.0 International License. Read Full License 
Construction and validation of a prognostic model for the assessment of postoperative overall survival of patients with metaplastic breast cancer: based on a retrospective large data analysis and Chinese multicenter study

Short title: a prognostic model for the assessment of postoperative overall survival of patients with metaplastic breast cancer

Ge Wang ${ }^{1}$, Xin Ren $^{1}$,Mengmeng Wang ${ }^{1}$, Xiaomin Sun ${ }^{1}$, Yongsheng Wang ${ }^{2}$,Shukun Zhang ${ }^{3}$, Jingy e $\mathrm{Li}^{4}$,Wenping $\mathrm{Lu}^{5}$,Baogang Zhang ${ }^{6}$,Pingping $\mathrm{Chen}^{7}$,Zhiqiang Shi ${ }^{2}$,Lijuan $\mathrm{Liu}^{8}$,Changgang Sun ${ }^{8,9^{*}}$, Jing zhuang ${ }^{8 *}$

1. Clinical Medical Colleges, Weifang Medical University, Weifang 261000, Shandong, P. R.China.

2. Shandong Cancer Hospital and Institute, Shandong First Medical University and Shandong Academy of Medical Sciences,Jinan 250000,Shandong, P. R.China.

3. Department of Pathology,Weihai Municipal Hospital,Cheeloo College of Medicine,Weihai 264200, Shandong University.

4. Department of Oncology,Linyi Central Hospital, Linyi 276000, Shandong, P. R.China.

5. Department of Oncology, Guang' anmen Hospital, China Academy of Chinese Medicine Sciences, Beijing 100053, China

6. Department of Pathology, Weifang Medical University, Weifang 261053, Shandong, P. R.China.

7. Department of Pathology,the people's hospital of Rizhao, Rizhao 276800,Shandong, P. R.China.

8. Department of Oncology, Weifang Traditional Chinese Hospital, Weifang 261000,Shandong, P. R.China.

9. Qingdao Academy of Chinese Medical Sciences, Shandong University of Traditional Chinese Medicine, Qingdao266112, Shandong, P. R. China.

*Correspondence: Changgang Sun , PhD, Prof

Department of Oncology, Weifang Traditional Chinese Hospital, 11166, Baotong East Street, High-tech Zone, Weifang City, Shandong Province;China. 
Qingdao Academy of Chinese Medical Sciences, Shandong University of Traditional Chinese

Medicine, No. 16369, Jingshi Road, Jinan City, Shandong Province,China.

E-mail: scgdoctor@126.com; $\quad$ Tel.: +86-536-8590-803.

*Correspondence: Jing Zhuang PhD

Department of Oncology, Weifang Traditional Chinese Hospital, No. 11166, Baotong East Street, High-tech Zone, Weifang City, Shandong Province;China.

E-mail: zhuangjing1226@163.com ; Tel.: +86-536-8590-803

\begin{abstract}
Purpose: Surgery is an important treatment for patients with metaplastic breast cancer (MBC). This study used prognostic clinicopathological factors to establish a model for predicting overall survival (OS) in patients with MBC.
\end{abstract}

Methods: Patients in the Surveillance, Epidemiology, and End Results (SEER) database diagnosed with MBC from 2010-2015 were selected and randomized into a SEER training cohort and an internal validation cohort. We identified independent prognostic factors after MBC surgery based on multivariate Cox regression analysis to construct nomograms. The discriminative and predictive power of the nomogram was assessed using Harrell's consistency index (C-index) and calibration plots. The decision curve analysis (DCA) was used to evaluate the clinical usefulness of the model.

Results: We divided 1044 patients from the SEER database randomly into a training set $(\mathrm{n}=732)$ and validation set $(\mathrm{n}=312)$ in a $7: 3$ ratio. Multifactorial analysis showed that age at diagnosis, $\mathrm{T}$ stage, $\mathrm{N}$ stage, $\mathrm{M}$ stage, tumor size, radiotherapy, and chemotherapy were important prognostic factors affecting OS. The C-index of nomogram was higher than the 7th edition of the AJCC TNM grading system in the SEER training set and validation set. The calibration chart showed that the survival rate predicted by the nomogram is close to the actual survival rate. The DCA showed that the nomogram is more clinically useful and applicable.

Conclusions: The prognostic model can accurately predict the post-surgical OS rate of patients with 
$\mathrm{MBC}$ and can provide a reference for doctors and patients to establish treatment plans.

Key words: metaplastic breast cancer, nomogram, overall survival, postoperative, multicenter

Abbreviations: metaplastic breast cancer (MBC); overall survival (OS); Surveillance, Epidemiology, and End Results Program (SEER); Harrell's consistency index (C-index); the World Health Organization (WHO); estrogen receptor (ER);progesterone receptor (PR); human epidermal growth factor receptor 2 (HER2); invasive ductal cancer (IDC); the American Joint Committee on Cancer (AJCC); hazard ratio (HR) ; confidence interval (CI); receiver operating characteristic curve (ROC); area under the curve (AUC); triple negative metaplastic breast cancer (TNMC); non-triple negative metaplastic breast cancer (NTNMC) ; decision curve analysis (DCA); interquartile range (IQR)

\section{Background}

Metaplastic breast cancer (MBC) is a rare subtype of breast cancer, with unique histopathological and molecular characteristics[1], accounting for less than $1 \%$ of all breast malignancies[2]. While most human cancers have only one histological component in a primary tumor, MBC is a heterogeneous cancer with diverse shapes and two or more different tissue types[3], including pure epithelial components (e.g., squamous cells), mesenchymal components (e.g., spindle cells, chondroids, osseous and myoid cells), or a mixture of the two[4]. In 2000, the World Health Organization (WHO) recognized $\mathrm{MBC}$ as a unique pathological entity[5]. Since then, the incidence of $\mathrm{MBC}$ has increased, which might be due to an increase in the number of cases, the improved recognition of the disease by pathologists, or both[6] .

MBC patients are not sensitive to chemotherapy and hormone therapy, and relevant targeted therapy and immunotherapy programs have not yet been developed. Therefore, surgical intervention is still the main treatment[7]. MBC is characterized by strong invasiveness, poor prognosis, and is often negative in estrogen receptor (ER), progesterone receptor (PR), and human epidermal growth factor receptor 2 (HER2) tests; its prognosis is worse than that of triple-negative breast cancer, and the survival rate is lower[8,9] .

At present, there are no standardized guidelines for MBC, and most clinicians still guide the treatment of MBC according to the scheme for invasive ductal cancer (IDC)[6]. However, compared with IDC, 
$\mathrm{MBC}$ has a larger tumor size, lower degree of differentiation, faster growth rate, and less axillary lymph node metastasis[4,10,11]. Since they have different clinicopathological features, it may be unreasonable to predict the pathological course and prognosis of $\mathrm{MBC}$ from existing studies on patients with IDC[12]. Therefore, MBC requires independent treatment and management strategies and its own customized model for personalized evaluation of postoperative survival of patients.

The TNM staging system proposed by the American Joint Committee on Cancer (AJCC) is a common tool used by oncologists to predict disease progression and design treatment strategies. However, considering that there are many factors affecting cancer progression, it may be unreasonable to predict the prognosis of MBC based on TNM staging alone[13]. In this respect, the nomogram makes up for its shortcomings[14]. Due to the low incidence of MBC, most of the studies on MBC come from a single medical institution or a limited cohort of case reports[12,15]. Therefore, based on data from the Surveillance, Epidemiology, and End Results (SEER) large-scale database in the United States, and the clinical and pathological data of patients with MBC from six large hospitals in Shandong Province, we explored the indicators that affect the prognosis of patients with MBC and constructed a model to predict survival. The objective is to provide a reference for informing the treatment of patients with MBC.

\section{Materials and methods}

\section{Patient selection and data processing}

Patient data from 2010-2015 were screened from the SEER database (1975-2016 varying) using SEER*Stat version 8.3.8 (https://seer.cancer.gov/). The obtained data were divided at random into a training set and an internal verification set in a 7:3 ratio. Based on clinical experience, existing literature, and the availability of information in the SEER data, the following variables were selected and evaluated: age, race, marital status (married: married/unmarried or domestic partner; single: unmarried; others: divorced/separated/widowed ), grade, tumor size, laterality, $\mathrm{T}$ stage, $\mathrm{N}$ stage (negative, positive), M stage, subtype, ER status, PR status, HER2 status, type of surgery (lumpectomy, mastectomy), radiation (no/unknown, yes), and chemotherapy (no/unknown, yes). The inclusion criteria for data screening were: (a) women with primary MBC, (b) MBC diagnosis was consistent with the International Classification of Disease for Oncology 3rd edition (coded as 8032/3, 8033/3, 8070/3, 
8570/3-8572/3, 8575/3, 8980/3, 8982/3), and (c) primary site: C50.0-C50.6, C50.8, or C50.9. The exclusion criteria were: (a) incomplete clinical pathological data or unknown records, (b) histological diagnosis was not positive, (c) survival time was less than 1 month or unknown, (d) patients with bilateral MBC, and (e) the patient did not undergo surgery at the primary tumor site.

For external verification, a Chinese multicenter validation set based on patients diagnosed between January 2010 and December 2020 from six hospitals (Shandong Cancer Hospital, Weihai Municipal Hospital, Rizhao People's Hospital, Linyi Central Hospital, Weifang Traditional Chinese Medicine Hospital, and the affiliated Hospital of Weifang Medical College) was included in the study. The inclusion/exclusion criteria for the Chinese multicenter data were consistent with that of the SEER dataset. Overall survival (OS) was used as the endpoint of this study and defined as the time from diagnosis to death of any cause or to the last follow-up. The last day of follow-up was December 1, 2020. For the retrospective analysis of the external validation set, we received approval from each institutional review board of the six institutions in Shandong, China. Since this was a retrospective study, patients were not required to sign an informed consent form. All patient data were used anonymously.

\section{Construction of nomogram}

In the training set, a multivariate Cox proportional hazard regression model was used to further analyze the prognostic variables identified from univariate analysis that were significantly related to postoperative OS in patients with MBC. The results are reported using hazard ratio (HR) and 95\% confidence interval (CI). Based on the results of multivariate analysis, a nomogram was constructed to predict the probability of OS at 1,3, and 5 years in patients with MBC.

\section{Discrimination and calibration of the nomogram}

We used the C-index, receiver operating characteristic curve (ROC), and area under the curve (AUC) to evaluate the distinguishing capabilities of the prediction model. A higher C-index value indicates a better discriminative performance by the nomogram. Calibration was evaluated such that how close the calibration curve was to the standard curve indicated the predictive ability of the model. To test the accuracy and reliability of the nomogram, the SEER internal verification set and Chinese multi-center 
external data set were used. To evaluate the effectiveness of the training and internal validation sets, the nomogram was compared with the 7 th edition AJCC staging model.

\section{Survival risk analysis and clinical usefulness of the nomogram}

The patients were divided into high-risk and low-risk groups according to the risk score obtained by the nomogram. The Kaplan-Meier method was used to assess the significance of survival differences between the two risk groups. The difference in survival between a triple negative metaplastic breast cancer (TNMC) group and a non-triple negative metaplastic breast cancer (NTNMC) group was also analyzed. Whether radiotherapy would bring survival benefits to elderly female patients was also studied. The DCA was used to evaluate the clinical usefulness of the nomogram.

\section{Statistical analysis}

All statistical analyses were performed using SPSS 25.0 (SPSS Inc., Chicago, IL, USA) and R software (version 4.0.3; http://www.r-project.org/). X-Tiles (version 3.6.1, Yale University, New Haven, CT, USA) was used to determine the best cut-off value for age and tumor size. Categorical variables were analyzed as frequencies and proportions. Cox proportional risk regression analysis was performed using SPSS. The nomogram, ROC curve, and calibration curve were generated using software packages ("foreign," "survival," "rms"); The DCA was performed using the R package ("stdca.R"). A P value $<0.05$ was considered statistically significant.

\section{Results}

\section{Patient characteristics}

As shown in Figure 1, 1044 patients with postoperative MBC were screened and divided into a training set $(n=732)$ and internal validation set $(n=312)$. As an external validation dataset, 40 eligible patients from a Chinese multicenter study were included. The best cut-off for age was 72 years, and the tumor size was 4.2, $6.7 \mathrm{~cm}$ (Fig.2). Table 1 summarizes the demographic and clinicopathological characteristics of the training, internal validation, and complete cohorts. In the training set, the median survival time was 31 months (interquartile range [IQR]: 18-54 months), and the median patient age was 61 years (IQR: 51-72 years). Among the patients, $47.4 \%$ were in the T2 stage, $76.9 \%$ were negative for lymph node metastasis, and $69.2 \%$ were diagnosed with TNMC. Table 1 shows the data 
distribution for the multiple centers in China. Among the patients, the median survival time was 41.5 months (IQR:_13-56.75 month), the median age was 52.5 years (IQR: 46-61 year), 47.5\% were at the T2 stage, $85.0 \%$ were negative for lymph node metastasis, and $80 \%$ had a mastectomy.

\section{Screening of prognostic factors and construction of nomogram}

Table 2 summarizes the results of univariate and multivariate Cox regression analyses in the training cohort. Univariate regression analysis showed that age at diagnosis, tumor size, marital status, grade, T stage, $\mathrm{N}$ stage, $\mathrm{M}$ stage, radiotherapy ,chemotherapy ${ }_{2}$ and type of surgery were significantly correlated with OS; while race, laterality, subtype, ER status, PR status, and HER2 status were not. Multivariate analysis identified that age at diagnosis, $\mathrm{T}$ stage, $\mathrm{N}$ stage, $\mathrm{M}$ stage, radiation, chemotherapy, and tumor size were independent predictors of survival. The significant variables obtained from multifactor analysis were used to construct a nomogram. Based on the prognostic factors selected from the training cohort, a nomogram was developed to predict the 1-, 3-, and 5-year survival probability of patients with MBC (Figure 3).

\section{Verification and evaluation of nomogram}

A good partition performance by the nomogram was verified. In the training cohort and validation cohort of SEER, the C-index of the nomogram was $0.803,0.769$, which was higher than that of the AJCC staging system $0.752,0.717$. In addition, the AUCs confirmed the superiority of the predictive model for predicting the 1-year, 3-year, and 5-year survival in the training cohort (nomogram vs. AJCC, 0.860 vs. $0.790 ; 0.811$ vs. $0.777 ; 0.827$ vs. 0.785 , respectively) and validation set (nomogram vs. AJCC, 0.721 vs. $0.689 ; 0.797$ vs. $0.750 ; 0.765$ vs. 0.734 , respectively) (Figure $4 \mathrm{a}-\mathrm{f}$ ). The discrimination and survival prediction performance of the training set and internal verification set were improved compared with the 7th edition of the AJCC TNM. The Chinese validation set C-index was 0.857 (95\%

CI, 0.726-0.988). The AUC values for 1 year, 3 years, and 5 years were $0.813,0.858$ and 0.881 , respectively (Figure 5a-c). The external verification set also proves that the nomogram has good distinguishing ability and predictive ability. In addition, the calibration curve test showed good agreement between the predicted values of the nomogram and the actual observed results (Figure 6a-c).

\section{Survival risk classification}


The Kaplan-Meier curve showed that there were significant differences in OS among the different risk subgroups of the SEER training set $(\mathrm{P}<0.001$, Fig. 7a, $)$, which was confirmed in two validation data sets (SEER validation set, $\mathrm{P}<0.001 \mathrm{Fig}$. $7 \mathrm{~b}$; multicenter validation set, $\mathrm{P}=0.0158$ Fig. $7 \mathrm{c}$ ). Among all 1044 patients, the nomogram showed great potential to differentiate between high- and low-risk groups ( $\mathrm{P}<0.001$, Fig. $7 \mathrm{~d})$. In addition, in the training cohort, a subgroup analysis was performed to determine whether MBC was triple negative or not and the results showed that the differences were not significant ( $\mathrm{P}=0.338$, Fig. 8a). Radiation therapy was found to provide a significant survival benefit in older women $(\mathrm{P}=0.0052$, Fig. $8 \mathrm{~b})$.

\section{Clinical application of the nomogram}

The 1-, 3-, and 5-year DCAs in the nomogram showed greater net benefits than either "full treatment" or "no treatment" and performed better than the AJCC stage model, which demonstrates potential clinical applicability of the nomogram (Figure 9a-c).

\section{Discussion}

At present, the most useful treatment for $\mathrm{MBC}$ is surgical resection. Due to the low incidence of $\mathrm{MBC}$, it is difficult to collect clinical and pathological data; therefore, we constructed a nomogram for predicting postoperative survival of MBC patients based on data from a large-scale database in the United States, and verified its performance using data from multiple Chinese centers. The AJCC staging system takes into account only tumor size and location, local lymph node invasion, and distant metastases[6], and its ability to predict postoperative survival in MBC patients is poor. The nomogram combines factors including age, tumor size, and treatment information to evaluate the prognosis of patients. As far as we know, this is the first nomogram for predicting the postoperative survival of patients with $\mathrm{MBC}$, and it can be used to provide patients with personalized services.

Compared with IDC, the OS rate of MBC was lower[16]. In this study, 7 factors were identified through univariate and multivariate analyses that were significantly related to the OS of patients with MBC, including age, $\mathrm{T}$ stage, $\mathrm{N}$ stage, $\mathrm{M}$ stage, tumor size, chemotherapy, and radiotherapy. $\mathrm{T}$ stage had the greatest impact on the survival of patients with MBC, and most patients had T2 stage disease.Many previous studies have also reported that the T stage of patients with MBC is higher than that of invasive ductal carcinoma. And compared with IDC, MBC is mostly blood metastasis. In addition, MBC is more likely to have lung and central nerve metastasis, while IDC has more bone 
metastasis[17]. There are great differences between MBC and IDC in clinicopathological and biological aspects[6]. The expression of $\mathrm{HG}$ and $\mathrm{Ki}-67$ in MBC is higher than that in IDC[4] . Therefore, it might not be appropriate to treat patients with $\mathrm{MBC}$ according to a management plan for IDC. This emphasizes the importance of the nomogram for informing personalized services for patients with MBC after surgery.

In this study, the type of surgery had nothing to do with the prognosis of MBC patients, which is consistent with previously reported results[18]. Whether it is lumpectomy or mastectomy, postoperative radiotherapy can bring survival benefits to patients with $\mathrm{MBC}[19]$. Li et al. showed that even elderly women ( $\geq 60$ years old) can benefit from radiotherapy[20]. We found that even older female patients ( $\geq 73$ years old), postoperative radiotherapy can also obtain significant survival benefits $(\mathrm{P}=0.0052)$. However, because these studies had small sample sizes or were retrospective, caution is still needed when treating patients with reference to these studies. The development of standardized radiotherapy guidelines based on prospective studies with sufficient sample size would be of great clinical value.

The differences between molecular typing of subtypes were not found to be statistically significant. When subtypes were grouped into TNMC and NTNMC groups, there was no significant difference in survival between the two groups $(\mathrm{P}=0.338)$. Previous studies have also shown this result[7] . In a retrospective analysis of $51 \mathrm{MBC}$ patients treated at Seoul National University Hospital, Kyu-Hyoung et al. found that triple-negative $\mathrm{MBC}$ is a favorable prognostic marker for patients with MBC. Their study also mentioned that TNMC is more prone to distant metastasis than NTNMC; however, after distant metastasis TNMC progresses more slowly, which may have caused the difference between the two subgroups. Since the study had a small sample of individual institutions, the mechanism behind this result needs to be further explored[21].

Histology of MBC contains 8 types after screening, including, metaplastic carcinoma, carcinosarcoma, squamous cell carcinoma, spindle cell carcinoma, sarcomatoid carcinoma, fibromatosis-like metaplastic carcinoma, low-grade adenosquamous carcinoma, and metaplastic carcinoma with chondroid differentiation or osseous differentiation[22,23]. When the data were grouped in a 7:3 ratio in this study, not all subtypes were included in the training set, and they were analyzed based on the whole population cohort. A univariate Cox regression analysis showed that histology was not a risk factor for OS after surgery in patients with $\operatorname{MBC}(\mathrm{p}=0.139)$. Previous studies also find no significant 
correlation between histology and prognosis[15,18]. Univariate analysis showed that marital status is a risk factor for the prognosis of MBC. Previous studies found that social environment also has an impact on this disease, which emphasizes that we might need to consider to the effect of non-biologic stressors on the disease in the future[7].

This study found that hormone receptor status (ER/PR) is not a risk factor that affects the survival of patients with MBC, which is consistent with the results of previous studies. Hormone therapy might not provide survival benefits to patients with $\mathrm{MBC}[6,16]$. While chemotherapy was found to provide a survival benefit in patients with MBC after surgery[24,25], it has a low impact. Studies have reported that most MBC patients have resistance to chemotherapy, including cancer cells undergoing the epithelial-to-mesenchymal transition and stem cell-like characteristics[26-29]. Joneja et al. used the first-generation gene sequencing method to compare $\mathrm{MBC}$, triple-negative breast cancer, and HER2-positive and hormone-positive breast cancer, and found that the expression rate of PD-L1 in MBC was higher than that of the other three cancer subtypes (46\% vs. $9 \%, 6 \%, 6 \%$, respectively; $\mathrm{p}<0.001)[30]$. Although the expression mechanism is not yet clear, this provides a theoretical basis for the treatment of MBC using the immune system[31]. Many mutated genes have been detected in MBC, and the most frequency mutated genes, TP53 and PIK3CA, suggest a direction for investigating targeted therapy[31-33].

Based on the results of the analysis, we constructed a nomogram to predict the postoperative survival of patients with MBC. The C-index and ROC curve of the nomogram performed better than the 7 th edition of the AJCC TNM staging system. The calibration curve shows that the 1-year, 3-year, and 5-year survival rates predicted by the nomogram are similar to the actual survival rates. The nomogram also has good clinical applicability. However, due to the rarity of the disease and the small amount of data in the external validation set, the external data set did not validate that the nomogram performs better than the AJCC stage. Nonetheless, since the 40 cases of MBC from the large hospitals in China are representative of the real-world MBC situation, the nomogram is suggested to be applicable for practical use.

The study has some limitations. First, some patients were excluded due to incomplete information when the data was screened, and some selection bias may exist. Second, some important parameters and specific information related to prognosis, such as the family history of breast cancer, vascular 
invasion, chemotherapy regimens, and targeted therapies, are missing from the SEER database. Third, this is a retrospective study based on SEER and needs to be validated in a prospective clinical trial.

\section{CONCLUSION}

In conclusion, the nomogram developed in this study is capable of predicting postoperative OS in patients with MBC. Validation of the internal dataset showed that the nomogram has good discrimination, predictive ability, and clinical practicability. The performance of the nomogram was further verified in the real world. These results suggest that the nomogram can help clinicians to predict postoperative OS in MBC patients and provide personalized services more accurately.

\section{Declarations}

\section{Funding}

No specific funding was disclosed.

\section{Conflict of Interest Statement}

All authors have no conflict of interest to declare.

\section{Data Availability Statement}

Publicly available datasets were analyzed in this study. This data can be found here: Surveillance, Epidemiology, and End Results (https://seer.cancer.gov/seerstat/software/). All data generated or analysed during this study are included in this article.

\section{Code availability}

Not applicable 


\section{Authors' contributions}

CGS and JZ designed and evaluated this study. YSW, SKZ, JYL, BGZ, PPC, and ZQS participated in collection materials of metaplastic breast cancer.GW, XR, WPL and LJL were involved in collating and evaluating data.MMW . XMS and CGS analyzed and interpreted the data. GW, XR, and JZ writing original draft and editing. GW, XR .MMW and XMS proofread the manuscript for important intellectual content. All authors contributed to data curation、 writing review and editing. All authors read and approved the final manuscript.

\section{Ethics approval and consent to participate}

For the Chinese multicenter dataset, the retrospective analysis of anonymous patient data was approved by the institutional review board at each participating institution.

\section{Consent to participate}

Not applicable.

\section{Consent for publication}

Not applicable.

\section{References}

1. Esbah O, Turkoz FP, Turker I, Durnali A, Ekinci AS, Bal O, Sonmez OU, Budakoglu B, Arslan UY, Oksuzoglu B. Metaplastic breast carcinoma: case series and review of the literature. Asian Pac J Cancer Prev. 2012;13(9):4645-4649.

2. Haque W, Verma V, Butler EB, Teh BS. Omission of radiotherapy in elderly women with early stage metaplastic breast cancer. The Breast. 2018;38:154-159.

3. Avigdor BE, Beierl K, Gocke CD, Zabransky DJ, Cravero K, Kyker-Snowman K, Button B, Chu D, Croessmann S, Cochran RL, Connolly RM, Park BH, Wheelan SJ, Cimino-Mathews A. Whole-Exome Sequencing of Metaplastic Breast Carcinoma Indicates Monoclonality with Associated Ductal Carcinoma Component. Clin Cancer Res. 2017;23(16):4875-4884.

4. Jung SY, Kim HY, Nam BH, Min SY, Lee SJ, Park C, Kwon Y, Kim EA, Ko KL, Shin KH, Lee 
KS, Park IH, Lee S, Kim SW, Kang HS, Ro J. Worse prognosis of metaplastic breast cancer patients than other patients with triple-negative breast cancer. Breast Cancer Res Treat. 2010;120(3):627-637.

5. Drekolias D, Mamounas EP. Metaplastic breast carcinoma: Current therapeutic approaches and novel targeted therapies. Breast J. 2019;25(6):1192-1197.

6. Pezzi CM, Patel-Parekh L, Cole K, Franko J, Klimberg VS, Bland K. Characteristics and treatment of metaplastic breast cancer: analysis of 892 cases from the National Cancer Data Base. Ann Surg Oncol. 2007;14(1):166-173.

7. Ong CT, Campbell BM, Thomas SM, Greenup RA, Plichta JK, Rosenberger LH, Force J, Hall A, Hyslop T, Hwang ES, Fayanju OM. Metaplastic Breast Cancer Treatment and Outcomes in 2500 Patients: A Retrospective Analysis of a National Oncology Database. Ann Surg Oncol. 2018;25(8):2249-2260.

8. Bae SY, Lee SK, Koo MY, Hur SM, Choi MY, Cho DH, Kim S, Choe JH, Lee JE, Kim JH, Kim JS, Nam SJ, Yang JH. The prognoses of metaplastic breast cancer patients compared to those of triple-negative breast cancer patients. Breast Cancer Res Treat. 2011;126(2):471-478.

9. El Zein D, Hughes M, Kumar S, Peng X, Oyasiji T, Jabbour H, Khoury T. Metaplastic Carcinoma of the Breast Is More Aggressive Than Triple-negative Breast Cancer: A Study From a Single Institution and Review of Literature. Clin Breast Cancer. 2017;17(5):382-391.

10. Bian T, Lin Q, Wu Z, Cui C, Qi C, Li L, Su X. Metaplastic carcinoma of the breast: Imaging and pathological features. Oncol Lett. 2016;12(5):3975-3980.

11. Nelson RA, Guye ML, Luu T, Lai LL. Survival outcomes of metaplastic breast cancer patients: results from a US population-based analysis. Ann Surg Oncol. 2015;22(1):24-31.

12. Han M, Salamat A, Zhu L, Zhang H, Clark BZ, Dabbs DJ, Carter GJ, Brufsky AM, Jankowitz RC, Puhalla SL, Johnson RR, Soran A, Steiman JG, McAuliffe PF, Diego EJ, Bhargava R. Metaplastic breast carcinoma: a clinical-pathologic study of 97 cases with subset analysis of response to neoadjuvant chemotherapy. Mod Pathol. 2019;32(6):807-816.

13. Chen S, Liu Y, Yang J, Liu Q, You H, Dong Y, Lyu J. Development and Validation of a Nomogram for Predicting Survival in Male Patients With Breast Cancer. Front Oncol. 2019;9:361.

14. Iasonos A, Schrag D, Raj GV, Panageas KS. How to build and interpret a nomogram for cancer prognosis. J Clin Oncol. 2008;26(8):1364-1370.

15. Leyrer CM, Berriochoa CA, Agrawal S, Donaldson A, Calhoun BC, Shah C, Stewart R, Moore HCF, Tendulkar RD. Predictive factors on outcomes in metaplastic breast cancer. Breast Cancer Res Treat. 2017;165(3):499-504. 
16. Paul Wright G, Davis AT, Koehler TJ, Melnik MK, Chung MH. Hormone receptor status does not affect prognosis in metaplastic breast cancer: a population-based analysis with comparison to infiltrating ductal and lobular carcinomas. Ann Surg Oncol. 2014;21(11):3497-3503.

17. Song Y, Liu X, Zhang G, Song H, Ren Y, He X, Wang Y, Zhang J, Zhang Y, Sun S, Liang X, Sun Q, Pang D. Unique clinicopathological features of metaplastic breast carcinoma compared with invasive ductal carcinoma and poor prognostic indicators. World J Surg Oncol. 2013;11:129.

18. Corso G, Frassoni S, Girardi A, De Camilli E, Montagna E, Intra M, Bottiglieri L, Margherita De Scalzi A, Fanianos DM, Magnoni F, Invento A, Toesca A, Conforti F, Bagnardi V, Viale G, Colleoni MA, Veronesi P. Metaplastic breast cancer: Prognostic and therapeutic considerations. J Surg Oncol. 2021;123(1):61-70.

19. Tseng WH, Martinez SR. Metaplastic breast cancer: to radiate or not to radiate? Ann Surg Oncol. 2011;18(1):94-103.

20. Li Y, Chen M, Pardini B, Dragomir MP, Lucci A, Calin GA. The role of radiotherapy in metaplastic breast cancer: a propensity score-matched analysis of the SEER database. J Transl Med. 2019;17(1):318.

21. Lim KH, Oh DY, Chie EK, Han W, Im SA, Kim TY, Park IA, Noh DY, Ha SW, Bang YJ. Metaplastic breast carcinoma: clinicopathologic features and prognostic value of triple negativity. Jpn J Clin Oncol. 2010;40(2):112-118.

22. Lois Dickie, Carol Hahn Johnson, Suzanne Adams, Serban Negoita. Solid Tumor Rules. July 2019 Update. 29 p.

23. Hennessy BT, Giordano S, Broglio K, Duan Z, Trent J, Buchholz TA, Babiera G, Hortobagyi GN, Valero V. Biphasic metaplastic sarcomatoid carcinoma of the breast. Ann Oncol. 2006;17(4):605-613.

24. Lien HC, Hsiao YH, Lin YS, Yao YT, Juan HF, Kuo WH, Hung MC, Chang KJ, Hsieh FJ. Molecular signatures of metaplastic carcinoma of the breast by large-scale transcriptional profiling: identification of genes potentially related to epithelial-mesenchymal transition. Oncogene. 2007;26(57):7859-7871.

25. Reddy TP, Rosato RR, Li X, Moulder S, Piwnica-Worms H, Chang JC. A comprehensive overview of metaplastic breast cancer: clinical features and molecular aberrations. Breast Cancer Res. 2020;22(1):121.

26. Zhang Y, Toy KA, Kleer CG. Metaplastic breast carcinomas are enriched in markers of tumor-initiating cells and epithelial to mesenchymal transition. Mod Pathol. 2012;25(2):178-184. 
27. Osako T, Horii R, Ogiya A, Iijima K, Iwase T, Akiyama F. Histogenesis of metaplastic breast carcinoma and axillary nodal metastases. Pathol Int. 2009;59(2):116-120.

28. Taube JH, Herschkowitz JI, Komurov K, Zhou AY, Gupta S, Yang J, Hartwell K, Onder TT, Gupta PB, Evans KW, Hollier BG, Ram PT, Lander ES, Rosen JM, Weinberg RA, Mani SA. Core epithelial-to-mesenchymal transition interactome gene-expression signature is associated with claudin-low and metaplastic breast cancer subtypes. Proc Natl Acad Sci U S A. 2010;107(35):15449-15454.

29. Shah DR, Tseng WH, Martinez SR. Treatment options for metaplastic breast cancer. ISRN Oncol. 2012;2012:706162.

30. Joneja U, Vranic S, Swensen J, Feldman R, Chen W, Kimbrough J, Xiao N, Reddy S, Palazzo J, Gatalica Z. Comprehensive profiling of metaplastic breast carcinomas reveals frequent overexpression of programmed death-ligand 1. J Clin Pathol. 2017;70(3):255-259.

31. Haque W, Teh BS. Current Practice and Future Directions for Metaplastic Breast Cancer. Ann Surg Oncol. 2018;25(Suppl 3):630-631.

32. Tray N, Taff J, Singh B, Suh J, Ngo N, Kwa M, Troxel AB, Chae YK, Kurzrock R, Patel SP, Sharon E, Denkert C, Ross JS, Adams S. Metaplastic breast cancers: Genomic profiling, mutational burden and tumor-infiltrating lymphocytes. Breast. 2019;44:29-32.

33. Gonzalez-Martinez S, Perez-Mies B, Carretero-Barrio I, Palacios-Berraquero ML, Perez-Garcia J, Cortes J, Palacios J. Molecular Features of Metaplastic Breast Carcinoma: An Infrequent Subtype of Triple Negative Breast Carcinoma. Cancers (Basel). 2020;12(7). 
Figures
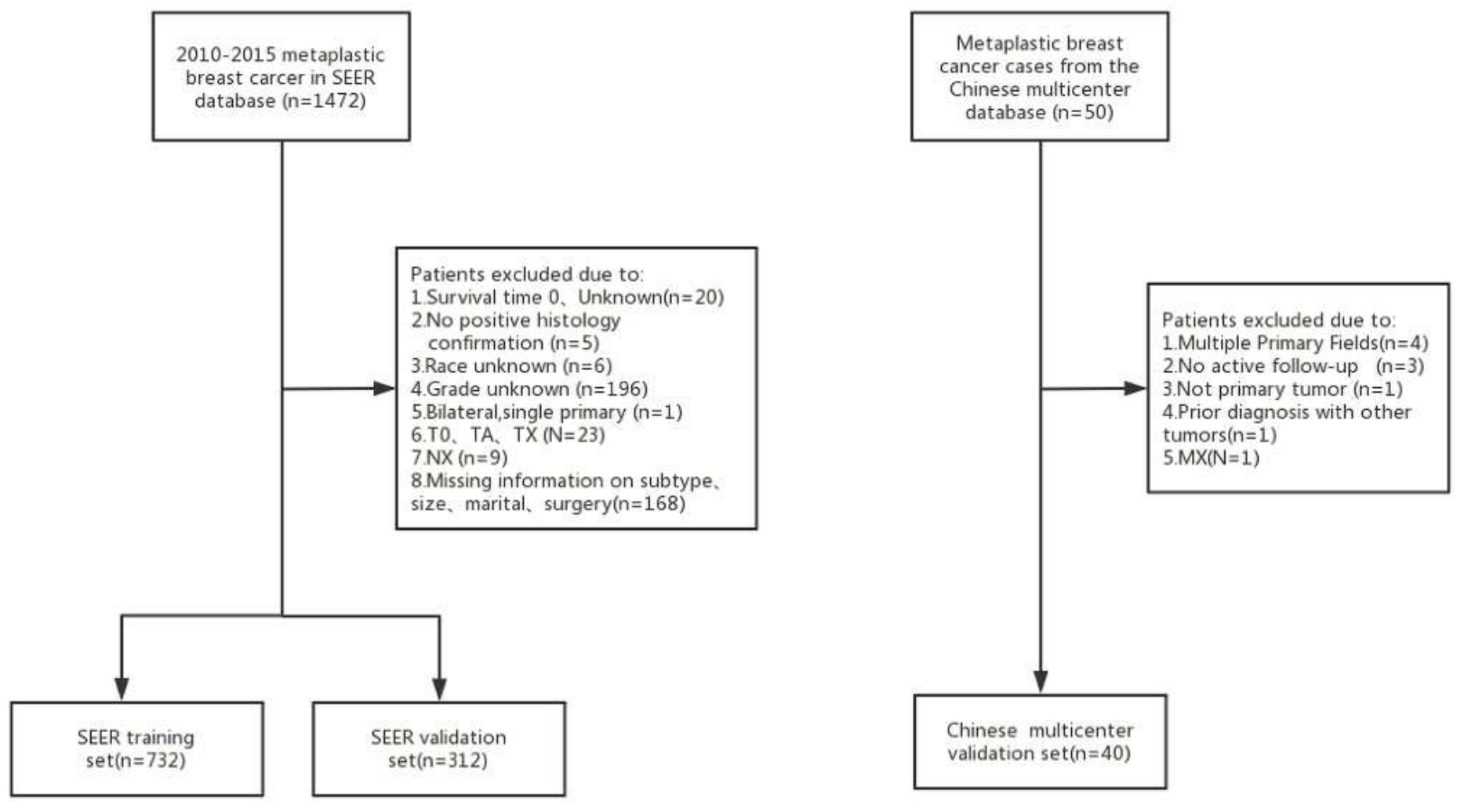

Figure 1

Flow diagram of MBC patient selection for the study. 


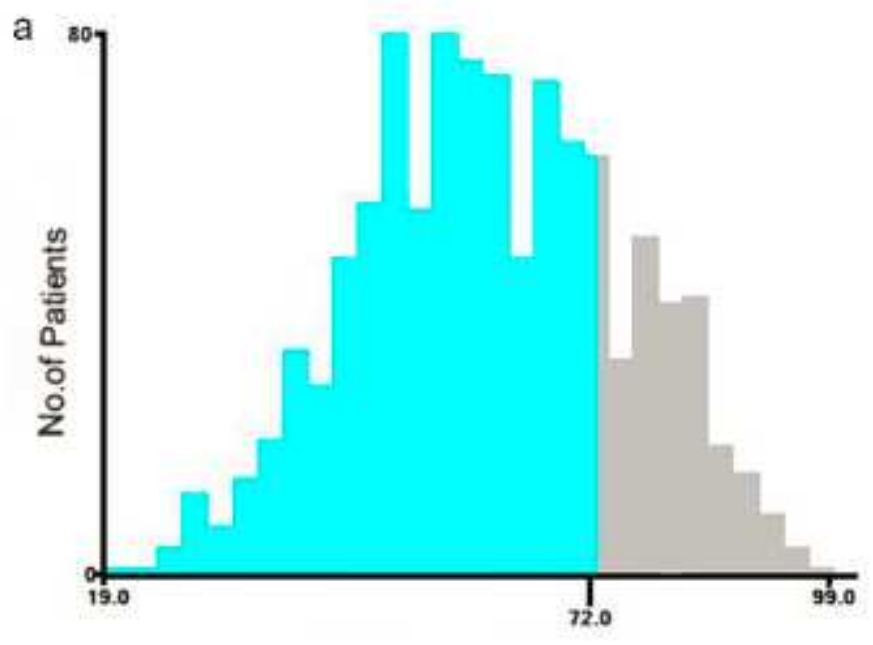

Age at diagnosis

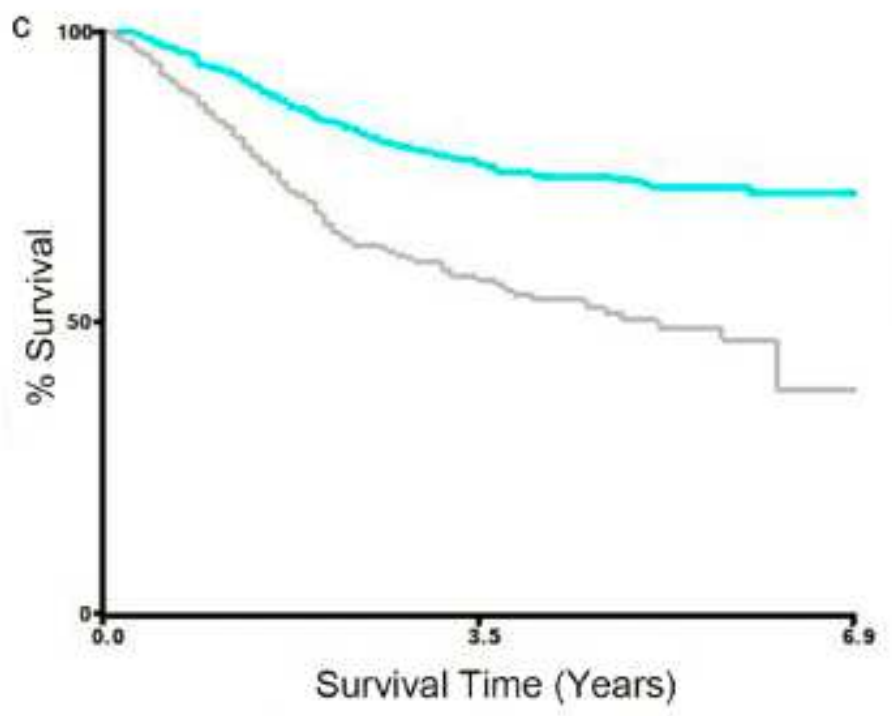

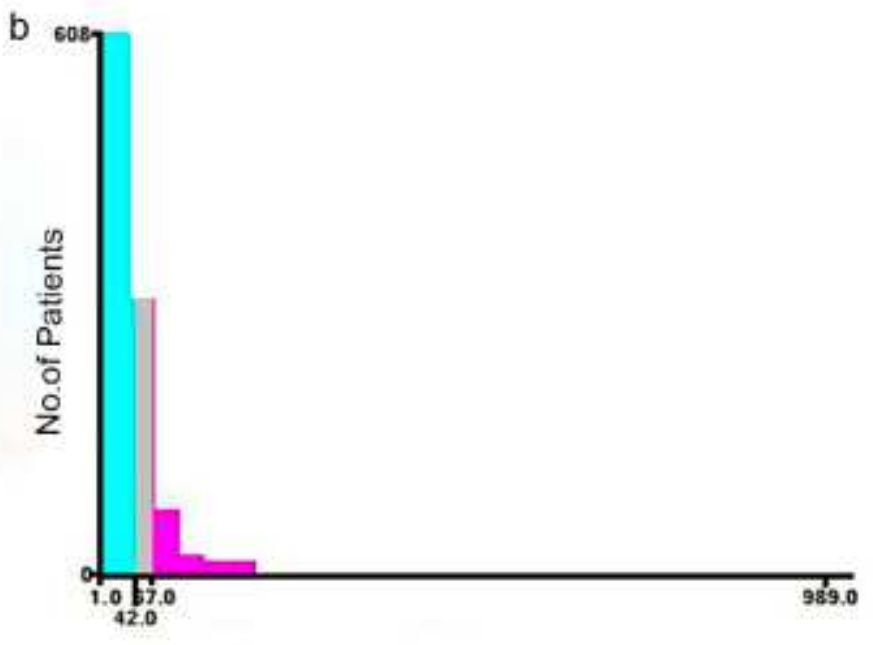

CS tumor size(2004-2015)

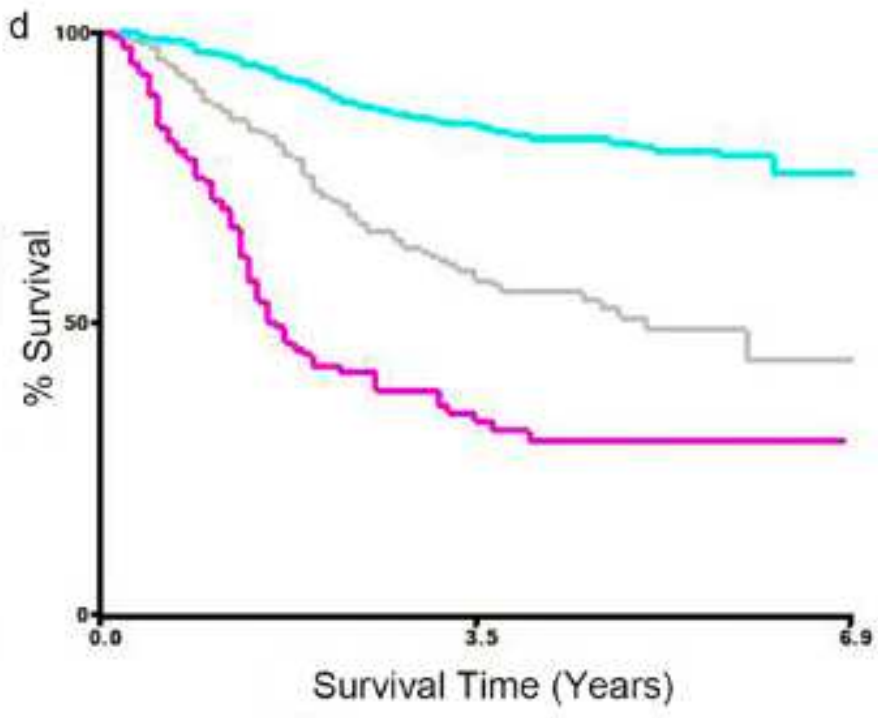

Figure 2

Identification of optimal cut-off values for age $(a, c)$ and tumor size $(b, d)$ by X-tile software analysis. The optimal cut-off value of age is identified as 72-years, and the cut-off for tumor size is identified as 4.2,6.7 $\mathrm{cm}$ based on entire cohort. 


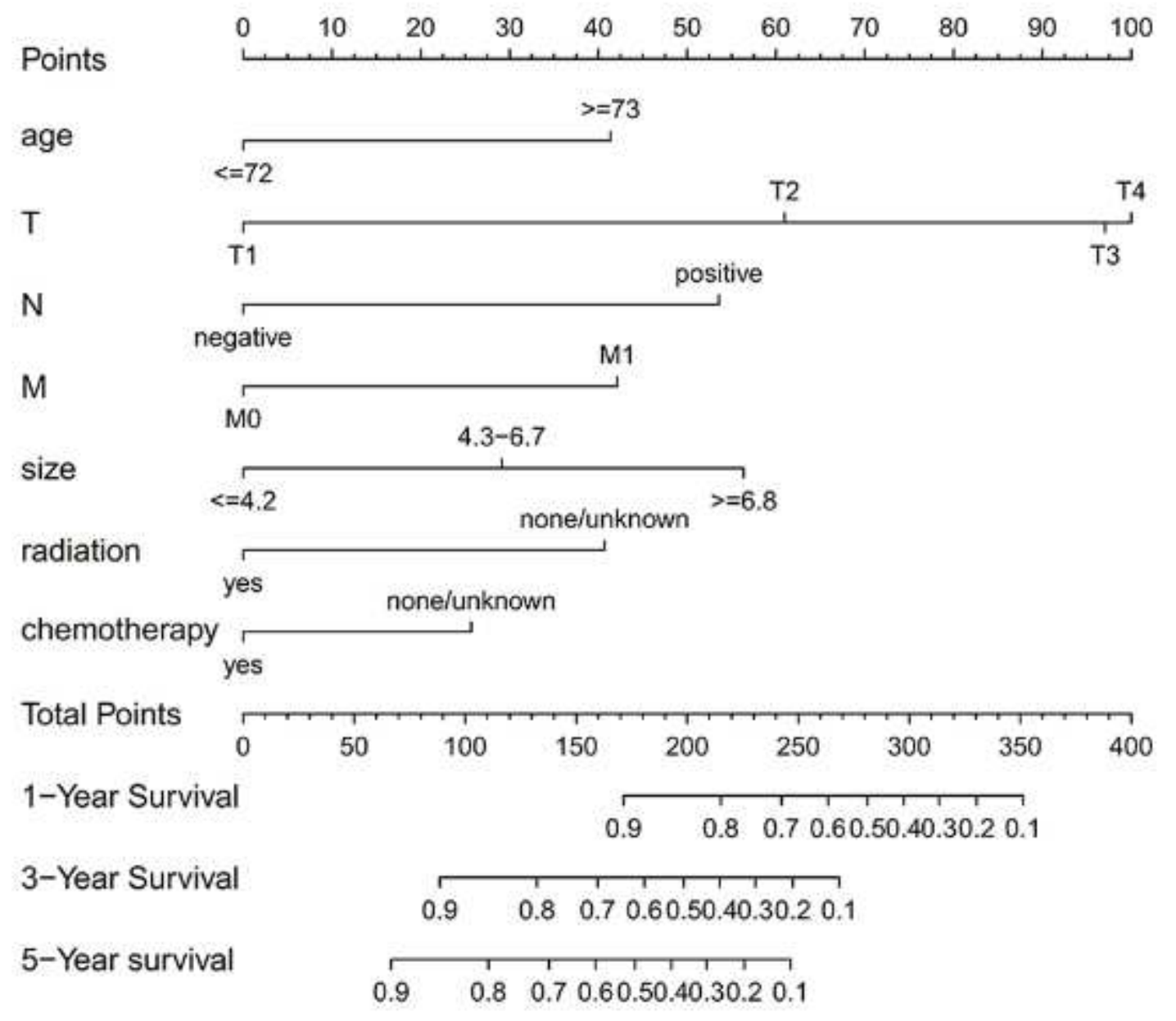

Figure 3

Nomograms for predicting the 1-, 3-, and 5-year OS rates of patients with MBC. The nomogram is applied by adding up the points identified on the scale of points for each variable. The OS rate is estimated by the total points for each individual. 


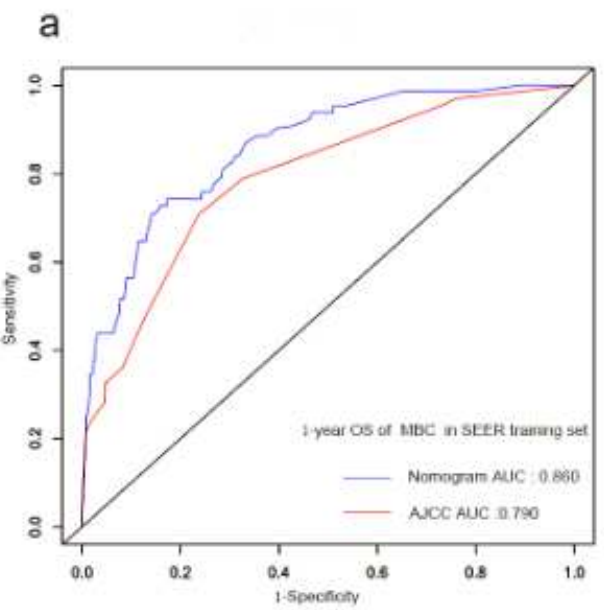

b

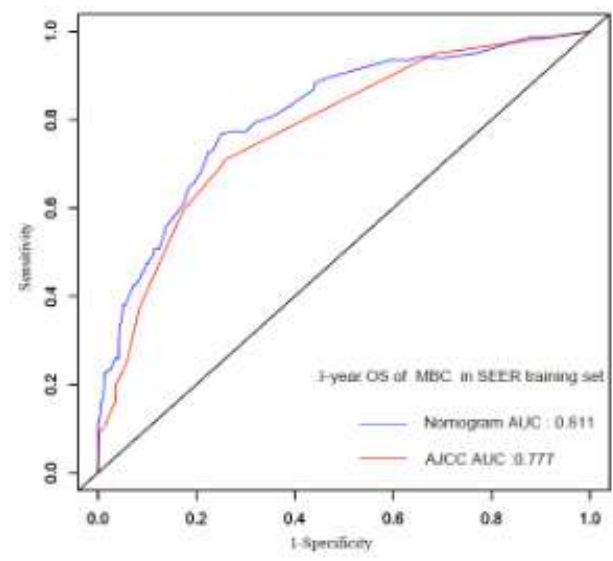

e

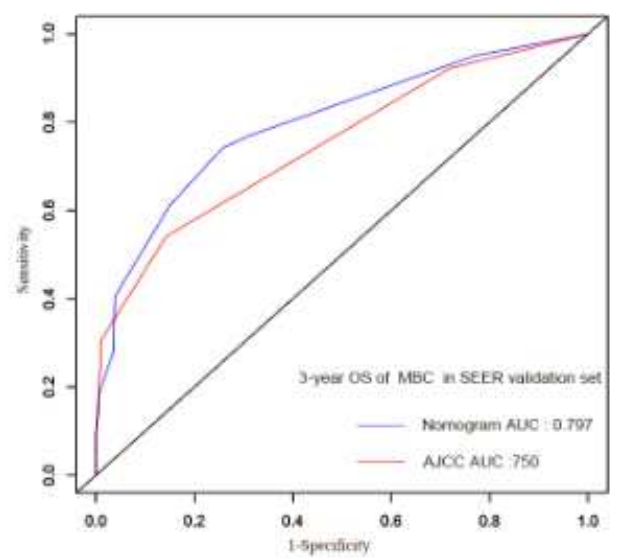

C
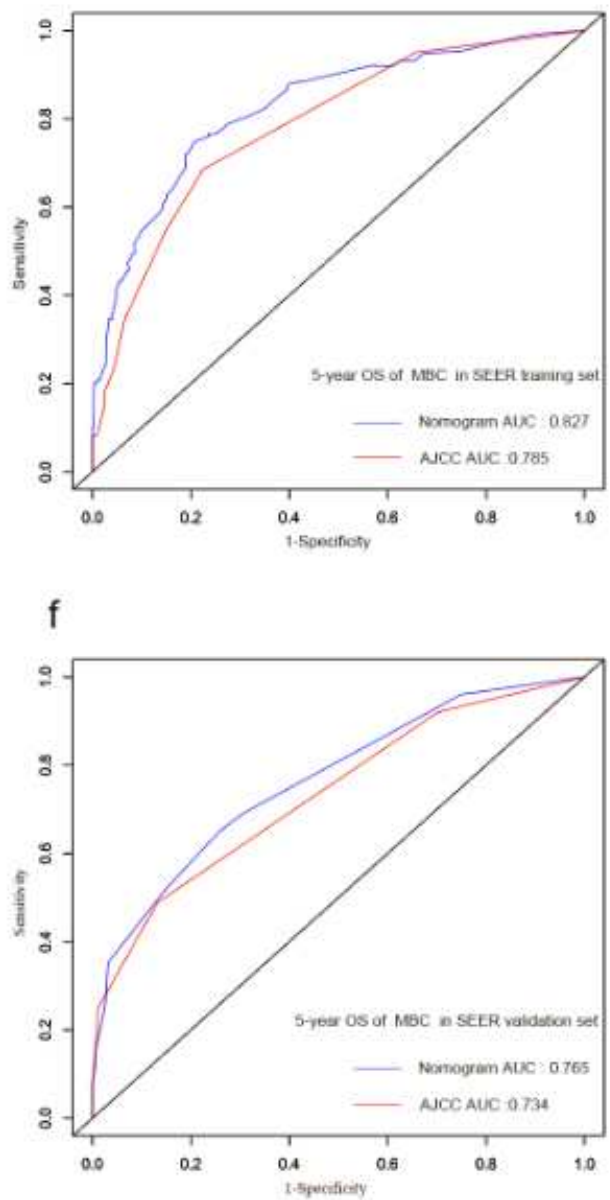

Figure 4

Comparison of the AUC of the nomograms and the 7th AJCC TNM staging system at 1, 3, and 5 years in the SEER training set $(a, b, c)$ and in the SEER validation set $(d, e, f)$. 

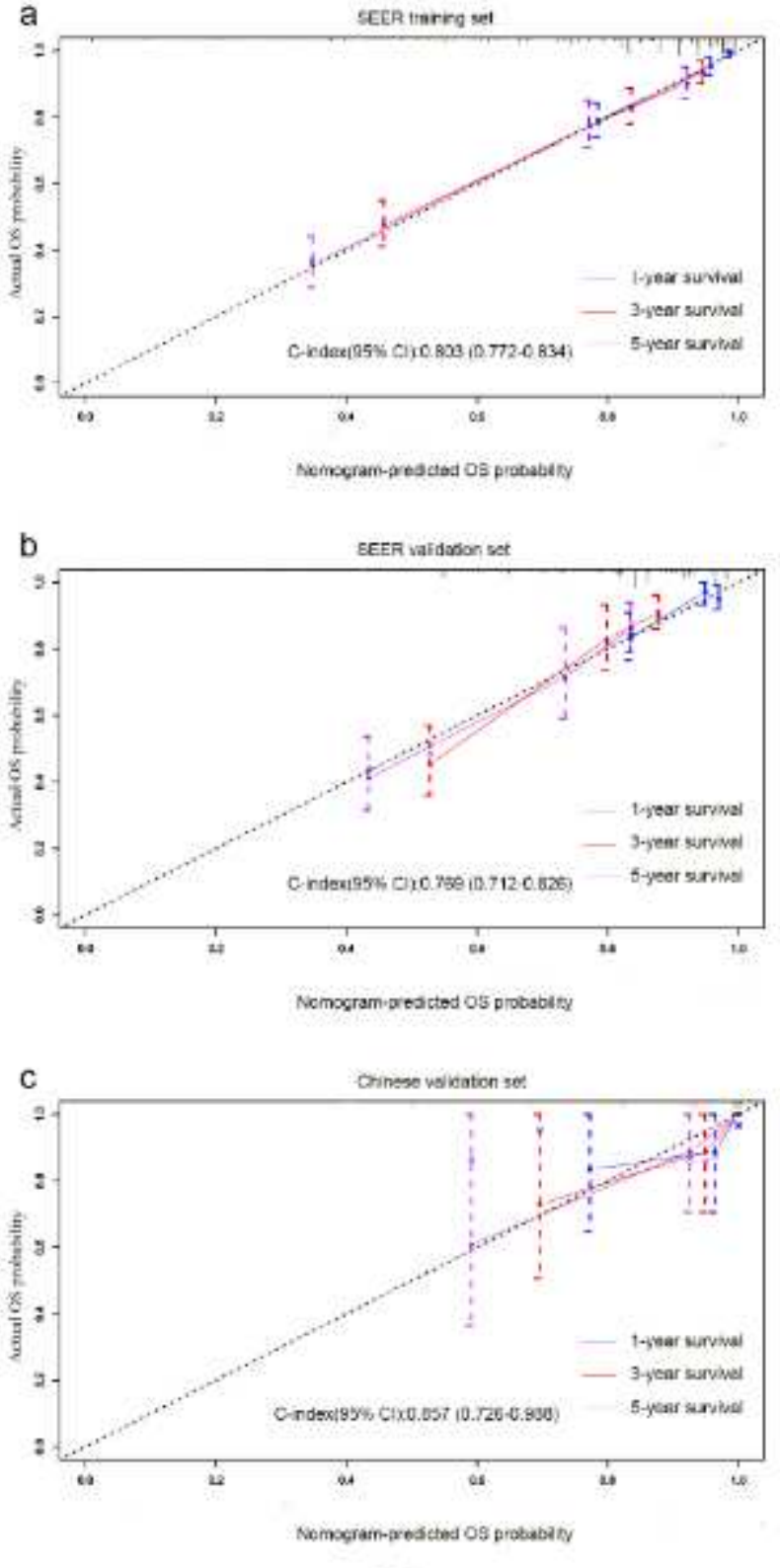

Figure 5

ROC curves and AUC for nomograms at 1, 3, and 5 years in the multicenter validation set (a, b, c). 

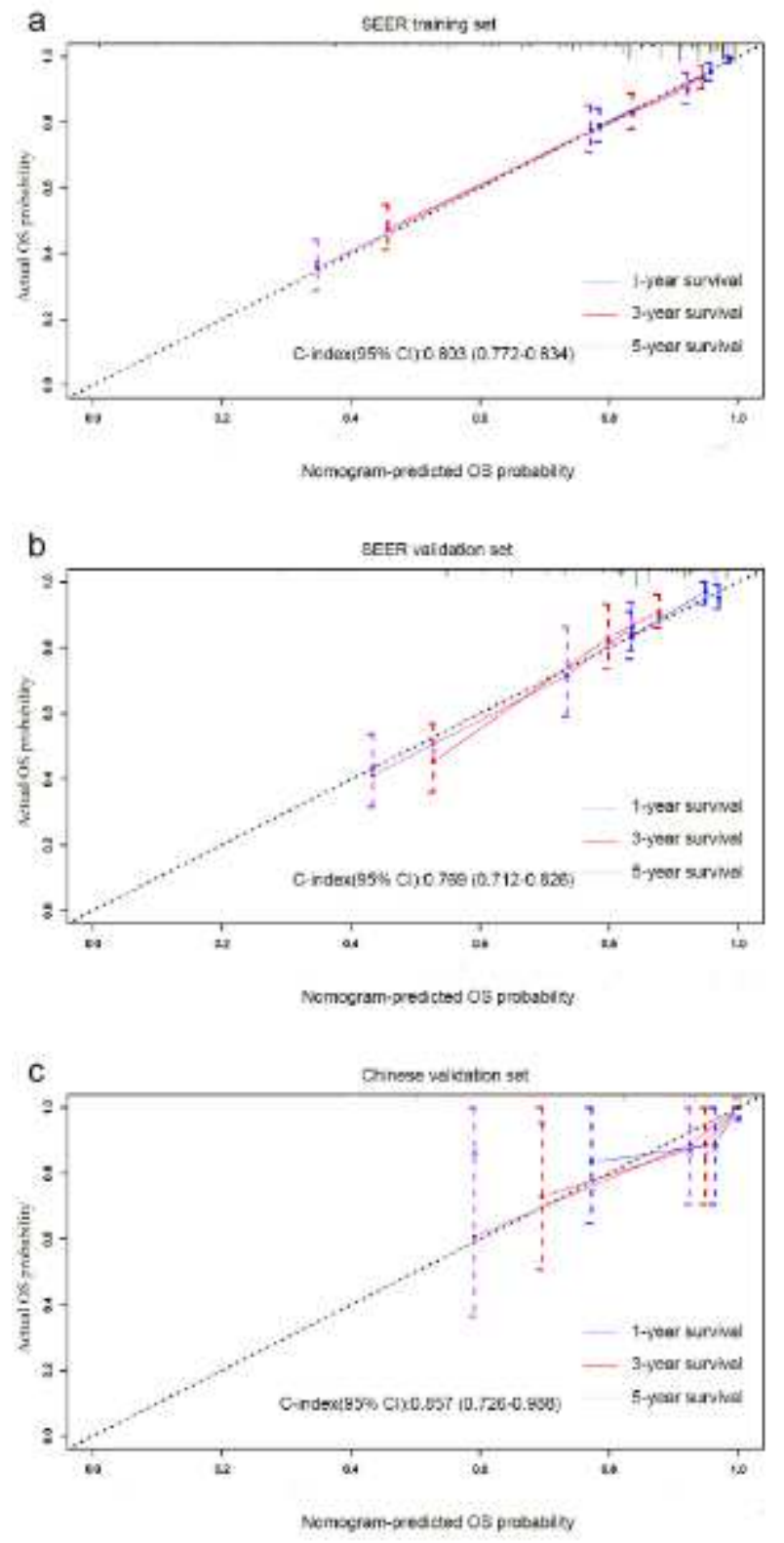

Figure 6

Calibration curves predicting the 1-, 3-, and 5-year OS of patients in the (a) training cohort, (b) SEER validation set, and (c) multicenter validation set. The $x$-axis plots the predicted survival probability, and the $y$-axis indicates the actual survival probability. The 45-degree inclined curve indicates that the predicted probability is in line with the actual probability. 

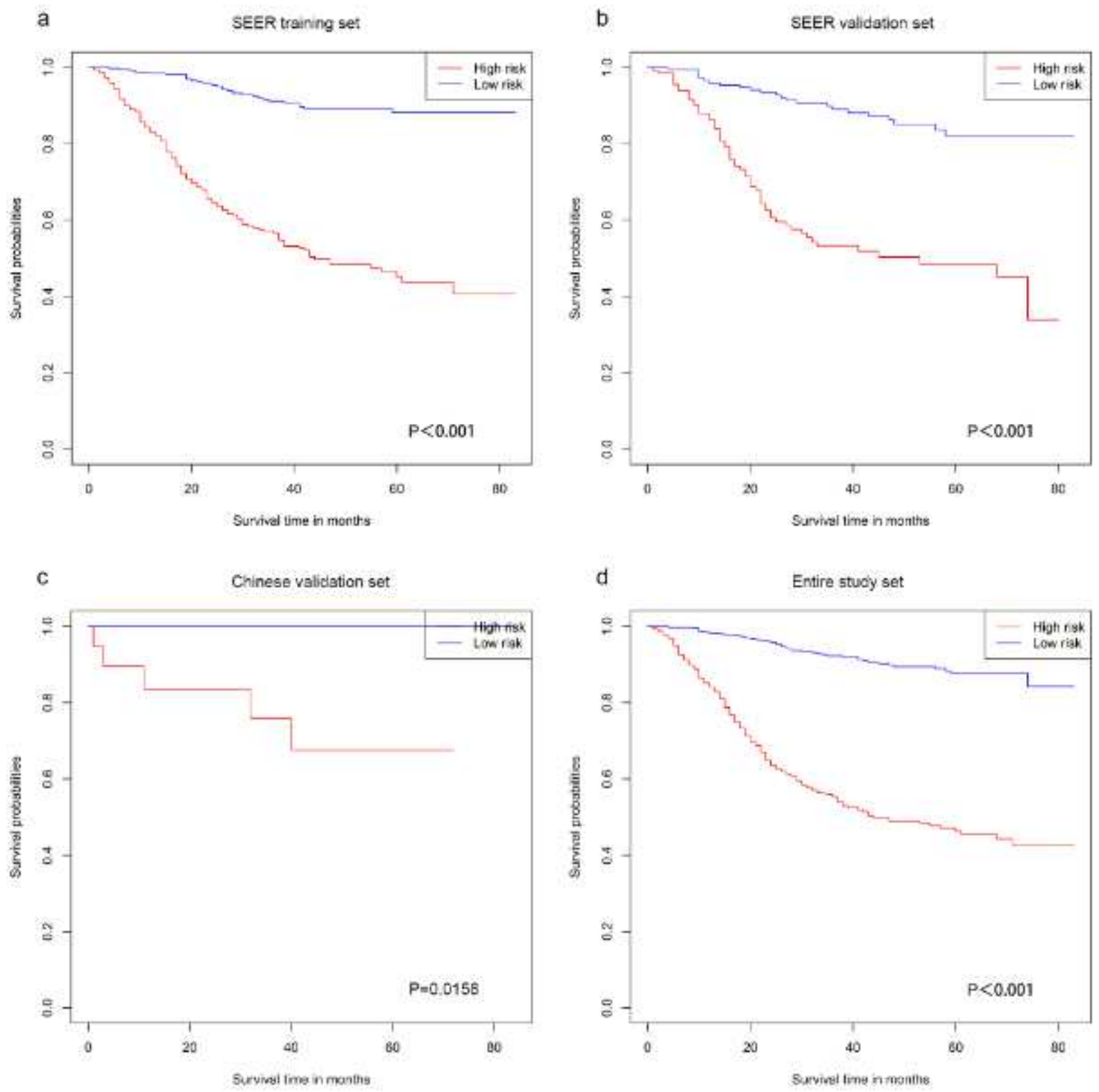

\section{Figure 7}

Kaplan-Meier survival curves of OS in the (a) SEER training set, (b) SEER validation set, (c) Chinese validation set, and (d) entire cohort of MBC patients. 

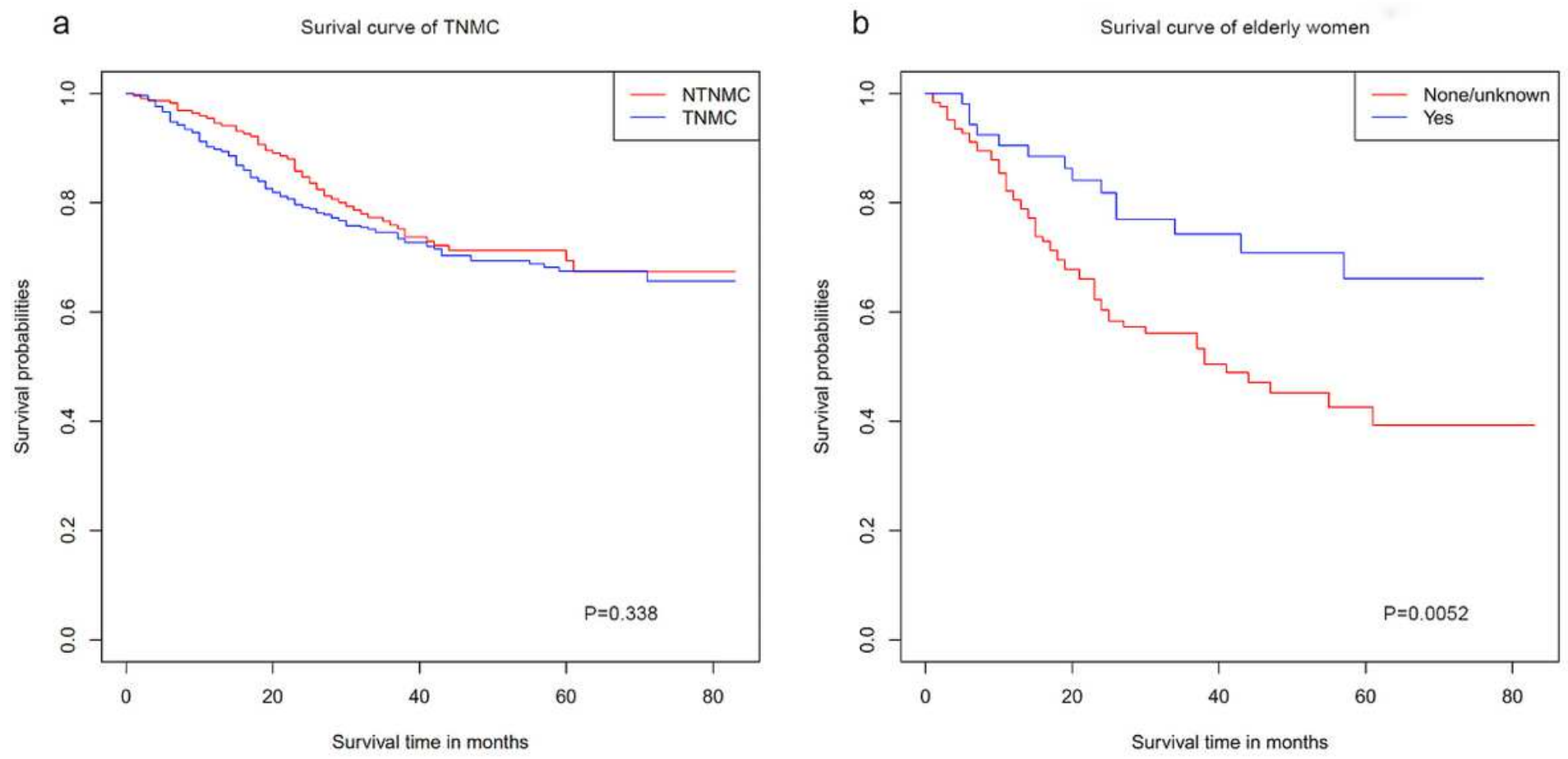

Figure 8

Kaplan-Meier curves of (a) OS for subtype stratification in the two groups and (b) OS for elderly women with adjuvant radiotherapy.

a

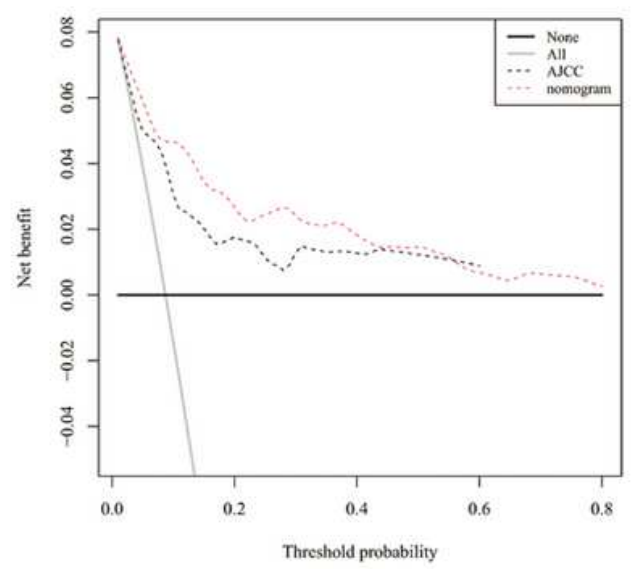

b

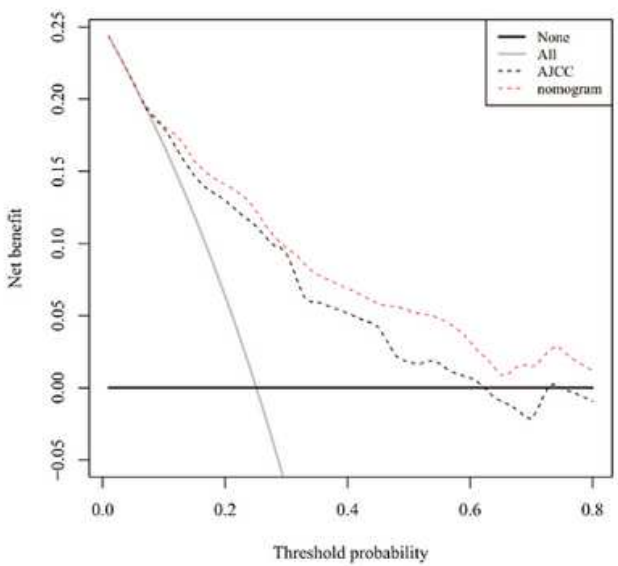

c

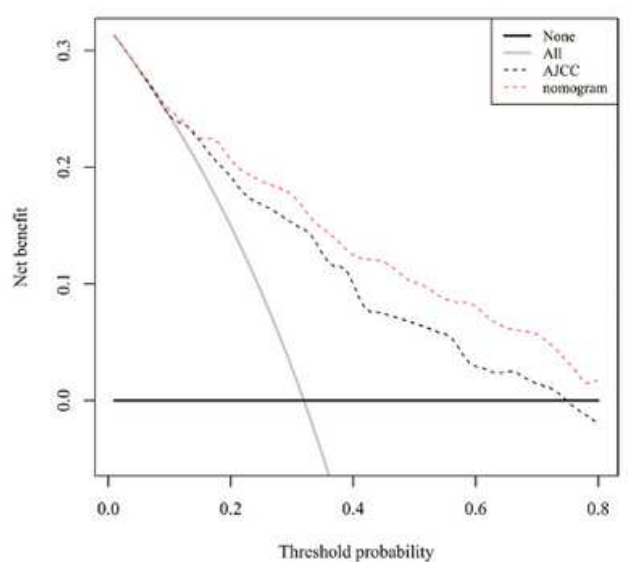

Figure 9

Decision curve analysis of the nomogram and AJCC stage in prediction of prognosis of MBC patients at the (a) 1-year, (b) 3-year, and (c) 5-year points in the training cohorts. 